\title{
Etude épidémio-clinique des références aux urgences pédiatriques du C.H.U Gabriel Touré
}

\author{
Clinical epidemiogical study of references to pediatric emergencies at Gabriel Toure teaching hospital
}

\author{
Dembélé $A^{1 *}$, Cissé $M E^{1}$, Diakité $A A^{1}$, Maïga $B^{1}$, Doumbia $A K^{1}$, Dembélé $M^{1}$, Coulibaly $O^{1}$, Togo $P^{1}$, Sacko $K^{1}$, Konaté $D^{1}$, Diall $H^{1}$, \\ Ahamadou $\mathrm{I}^{1}$, Sylla $\mathrm{M}^{1}$, Dicko $\mathrm{F}^{1}$, Sidibé $\mathrm{LN}^{1}$, Togo $\mathrm{B}^{1}$, Coulibaly $\mathrm{YA}^{1}$, Diakité $\mathrm{FL}^{1}$, Koné $\mathrm{Y}^{1}$
}

1. Département de Pédiatrie du CHU-Gabriel Touré

*Auteur correspondant : Email : adamadembele2206dochgt@yahoo.fr, Département de pédiatrie, CHU Gabriel Touré

\section{Résumé}

Introduction : Le système national de soins au Mali peut être schématisé sous la forme d'une pyramide dont la base représente le Centre de Santé Communautaire (CSCom) pour les soins primaires, le Centre de Santé de Référence (CSRef) constitue le premier niveau de référence suivi des hôpitaux régionaux et au sommet, les hôpitaux nationaux ou (dernier recours médical du pays). L'objectif de notre étude était d'étudier les aspects épidemio-cliniques des références aux urgences pédiatriques du CHU Gabriel Touré. Matériel et méthodes : Nous avons réalisé une étude prospective transversale de Janvier à Juin 2017, soit une période de 6 mois. Elle a concerné les enfants âgés de 1 mois à 15 ans référés aux urgences pédiatriques du $\mathrm{CHU}$ Gabriel Touré quel que soit le motif. L'enquête a été menée par interview des mères ou accompagnantes de l'enfant à leur admission avec une fiche d'enquête après un consentement obtenu au préalable. Résultats : Pendant notre période d'étude, 234 enfants ont été référés sur un total de 565 enfants hospitalisés aux urgences, soit une fréquence de $41,4 \%$. Le sex-ratio était de 1,9 et les nourrissons de moins de 12 mois étaient les plus concernés avec $51,3 \%$ des cas. Pour la majorité des cas $(126 / 234)$, les patients référés venaient des CSRéf soit $53,8 \%$. La dyspnée était le motif de référence le plus fréquent (105 cas/234) suivie de la fièvre (72 cas). C'est pendant les heures de garde (17heure-08heure) que la majorité des patients référés ont été reçu aux urgences soit $59 \%$. Le paludisme était la pathologie de référence la plus fréquente $(29,4 \%$ des cas) suivi des pathologies broncho-pulmonaires, 17,1\%. La durée d'hospitalisation était de 3 jours dans $28,6 \%$. Le taux de létalité était de12, $39 \%$. Conclusion : Malgré des multitudes de structures de santé, une pyramide sanitaire bien codifiée, la référence reste faible dans notre pays.

Mots clés: référence, urgence, pédiatrie.

\footnotetext{
Abstract

Introduction: The national health care system in Mali can be schematized in the form of a pyramid whose base represents the Community Health Center (CSCom) for primary care, the Reference Health Center (CSRef) constitutes the first level of reference followed. regional hospitals and at the top, national hospitals or (last medical resort in the country). The objective of our study was to
}

study the epidemiological and clinical aspects of referrals to pediatric emergencies at the Gabriel Toure Teaching Hospital. Material and methods: We carried out a crosssectional prospective study from January to June 2017, i.e. a period of 6 months. It concerned children aged 1 month to 15 years referred to the pediatric emergencies of the Gabriel Toure Teaching Hospital whatever the reason. The survey was conducted by interviewing mothers or those accompanying the child on admission with a survey sheet after consent obtained beforehand. Results: During our study period, 234 children were referred out of a total of 565 children hospitalized in the emergency room, a frequency of $41.4 \%$. The sex ratio was 1.9 and infants under 12 months were the most affected with $51.3 \%$ of cases. For the majority of cases (126/234), the patients referred came from the CSRref, ie $53.8 \%$. Dyspnea was the most frequent reference reason (105 cases / 234) followed by fever (72 cases). It was during on-call hours ( 5 p.m. to 8 a.m.) that the majority of referred patients were seen in the emergency room, i.e. $59 \%$. Malaria was the most frequent reference pathology $(29.4 \%$ of cases) followed by bronchopulmonary pathologies, $17.1 \%$. The length of hospital stay was 3 days in $28.6 \%$. The case fatality rate was $12.39 \%$. Conclusion: Despite multitudes of health structures, a well-codified health pyramid, the reference remains low in our country.

Keywords: referral, emergency, pediatrics.

\section{Introduction}

Les urgences pédiatriques constituent l'ensemble des états morbides qui, à une plus ou moins brève échéance, menacent la vie de l'enfant et en nécessitent une prise en charge rapide et adéquate [1]. Dans les pays en voie de développement particulièrement en Afrique subsaharienne les problèmes sanitaires sont encore caractérisés par la persistance des maladies épidémiques dans un contexte économique défavorable. Cette situation oblige les responsables des services de santé à faire des choix mettant en première ligne la santé publique et les soins de santé primaire [2]. Dans ces pays, l'urgence pédiatrique relève souvent d'une " médecine en catastrophe " et plusieurs études montrent l'extrême gravité des affections vues dans des consultations d'urgence [3].

La mortalité liée aux urgences est importante : elle est de $26,1 \%$ dans le service de pédiatrie du C.H.U Gabriel TOURE au Mali [1]. La politique sanitaire du Mali est conforme d'une part aux grands principes de l'Organisation Mondiale de la Santé (OMS) dont il est membre et d'autre part à ses réalités socio-économiques 
et culturelles. Cette politique a mis en place un système national de soins qui peut être schématisé sous la forme d'une pyramide dont la base représente le Centre de Santé Communautaire (C.S.Com) pour les soins primaires, le Centre de Santé de Référence (CSRef) constitue le premier niveau de références suivi des hôpitaux régionaux et au sommet, les hôpitaux nationaux ou (dernier recours médical du pays) [4]. Vu la fréquence élevée de la morbidité infantile, il est important de chercher à savoir si les références évacuations sont correctement menées selon cette pyramide et surtout quelles sont les affections référées aux urgences pédiatriques du C.H.U Gabriel Touré.

\section{Matériel et méthodes \\ Cadre d'étude}

Notre étude s'est déroulée dans le département de pédiatrie du CHU - Gabriel Touré de Bamako. Situé au centre de la ville, ce département reçoit les patients de toutes les communes de Bamako et ceux référés par les autres localités du Mali. Malgré l'existence des centres de santé communautaires et les centres de santé de référence, l'affluence y reste encore très élevée. II est composé d'un service de néonatologie auquel est rattachée I'URENI (Unité de Récupération et d'Education Nutritionnelle Intensive), un service de pédiatrie générale et un service des urgences pédiatriques. Le sevice des urgences pédiatriques a été créé en 2010 dans le cadre de la restructuration du département. II comporte un hall d'accueil qui est aussi une salle de tri, deux salles de consultation, un secteur d'hospitalisation de 20 berceaux et 6 lits avec deux salles pour les nourrissons et une salle pour les grands enfants.

Type et période d'étude

II s'agit d'une étude prospective, descriptive et transversale qui s'est déroulée du 1er Janvier au 30 Juin 2017, soit une période de 6 mois.

Critères d'inclusion: Nous avons inclus tous les nourrissons et les enfants de 1 mois à 15 ans référés aux urgences pédiatriques du CHU Gabriel Touré quel que soit le motif de la référence.

Collecte des données : Un questionnaire comportant différents éléments a été élaboré pour recueillir les données. Les variables étudiées étaient entre autres : l'identification des patients et leurs parents (pères et mères), la référence (l'heure d'admission, mode d'admission), l'examen clinique, la prise en charge et le devenir des patients.

Saisie et analyse des données : La saisie et l'analyse des données ont été effectuées sur le logiciel SPSS Statistics viewer version 23.

Considérations éthiques : Un consentement éclairé individuel verbal a été recherché et obtenu chez les parents ou accompagnants des malades, avant toute inclusion.

Nous avons été confrontés à des difficultés relatives à une précision sur l'âge des parents, le traitement reçu antérieurement et le déroulement de la symptomatologie.

\section{Résultats}

Au cours de notre étude, nous avons pu colliger 234 patients remplissant les critères d'inclusion. Les enfants de moins de 12 mois étaient les plus fréquents $(51,3 \%)$ (tableau I). Le sexe masculin était le plus représenté avec $66 \%$ des cas soit un sex-ratio de 1,9 (tableau I). La majorité des pères étaient des ouvriers $(81 / 234)$ soit $34,6 \%$ et ils étaient non scolarisés dans $58,8 \%$ des cas (tableau I). Les mères des patients étaient majoritairement femmes au foyer, soit $85 \%$ et étaient non scolarisées dans $62,4 \%$ (tableau I).

Avec $53,8 \%$, les CSRéf constituaient les structures qui ont majoritairement référées aux urgences pédiatriques du CHU GT et la dyspnée était le motif de référence le plus fréquent avec $44,8 \%$ des cas (tableau II).

La majorité des patients soit $59 \%$ ont été admis entre 17 heures-08heures contre $41 \%$ admis entre 08heures16 heures.

Le transport en commun était le moyen de déplacement le plus fréquemment utilisé par les patients (figure 1).

Le paludisme grave était le diagnostic le plus fréquemment évoqué suivi des pathologies bronchopulmonaires avec respectivement $27,7 \%$ et $19,2 \%$ des cas (tableau III)

La quasi-totalité des patients (216/234) a reçu de l'antibiotique et l'association ceftriaxone + gentamycine injectable était la plus fréquemment utilisée, $31,6 \%$ (tableau IV). Dans $43 \%$ des cas les malades avaient reçu des antipaludiques (tableau IV).

La durée d'hospitalisation était de 3 jours dans $28,6 \%$ des cas.

Dans la majorité des cas (82,9\%), les patients sont guéris sans séquelles. Néanmoins nous avons déploré $12,4 \%$ de décès. Le paludisme grave a été la pathologie la plus létale avec 6 décès sur 24 (figure 3).

\section{Discussion}

Pendant notre période d'étude, du 1er Janvier au 30 Juin 2017, 565 enfants ont été hospitalisé aux urgences parmi lesquels, 234 étaient référés soit une fréquence de 41,4\%. Les enfants âgés de moins de 12 mois étaient les plus représentés, soit $51,3 \%$. Notre résultat est discutable avec ceux d'autres auteurs : $[5,6,7,8]$ qui ont rapporté une prédominance de la tranche d'âge ( 0 à 5 ans). Ce phénomène s'explique naturellement par la vulnérabilité des enfants à cet âge, et leur sensibilité aux infections. Le sexe masculin était majoritaire, soit $66 \%$. Cette prédominance avait été relevée dans des études précédentes cependant aucune des études n'a pu établir un lien formel entre le sexe et la référence. Le niveau d'instruction et la profession des parents semblent avoir une incidence concrète sur le mode de vie et la survenue de manifestations morbides chez leurs enfants. Ainsi, dans notre étude, nous avons constaté que les parents des enfants appartenaient, pour la plupart, à des classes sociales peu favorisées économiquement et peu scolarisées. En effet, les pères ouvriers et non scolarisés y étaient les plus nombreux. De même, les femmes au 
foyer et non scolarisées y étaient plus représentées. Ces résultats sont superposables à ceux qui se trouvent dans des études précédentes comme celles d'Abdou $\mathrm{R} O$ [1] et de Sy $O$ [8]. La majorité des patients, venaient des CSRéf soit $53,8 \%$. Ce résultat est encourageant et démontre le respect de la pyramide sanitaire par la population malgré que la fréquence globale des références soit faible ( $41,4 \%$ des hospitalisés). II peut être amélioré par une communication pour le changement de comportement.

La plupart de nos patients ont été admis de 17heure08heure (heure de garde), soit $59 \%$ de l'effectif et les transports en commun ont été les moyens de déplacement les plus utilisés pour rejoindre l'hôpital. Ce constat s'explique peut-être par le fait que le déplacement des ambulances est payant (3000F CFA $=5 \$$ US) et la précarité des parents. Nos résultats sont superposables à ceux de Coulibaly M Z qui trouva $73,8 \%$ d'admission entre $14 \mathrm{H} 30-00 \mathrm{H}$. Seulement $34 \%$ de nos patients avaient reçu un traitement antérieur prouvé, notre résultat est inférieur à celui de Bobossi Seringne B en Bangui [9] qui a rapporté $89,4 \%$ de traitement antérieur avant l'admission.

La dyspnée était le motif de référence le plus fréquent soit (105 cas) suivi de la fièvre (72 cas), les convulsions (37 cas) et l'anémie (33 cas). La prédominance de ces symptômes pourrait s'expliquer par la fragilité particulière du nourrisson face aux infections. Les références sont de causes diverses et variées. Ainsi, dans notre étude, les pathologies les plus rencontrées étaient : le paludisme grave $27,7 \% \quad(\mathrm{~N}=65)$, les pathologies bronchopulmonaires $19,2 \% \quad(\mathrm{~N}=45)$, les cardiopathies $14,1 \%$ $(\mathrm{N}=33)$, méningite 12,8\% $(\mathrm{N}=30)$. Avec $27,7 \%$ de paludisme. Notre résultat est comparable à ceux de TRAORE A [5] et DAFFE H [10] qui ont montré que le paludisme grave était la première étiologie des urgences pédiatriques. Au Bénin, également le paludisme grave constitue la première cause des urgences avec $56 \%$ des cas selon Dan $V$ et coll. [11] et la déshydratation par diarrhée constituait la troisième cause des urgences médicales pour $10 \%$ des cas [12]. Au Congo, Atanda H L et coll. ont trouvé des résultats identiques soit $11,50 \%$ [13]. Ces résultats confirment que le paludisme reste, encore, la première cause de morbidité chez les enfants en milieu hospitalier au Mali voire dans la sous-région. En fonction de l'étiologie, 92,3\% des patients ont été traités par antibiotique parmi lesquels $31,6 \%$ ont reçu une bithérapie (ceftriaxone + gentamycine). Le traitement antipaludique a été institué chez $43 \%$ des patients, totalement composé d'artésunate injectable. La durée moyenne de séjour dans le service était de 3 jours, identique à celle de DAFFE $H$ [10] qui avait trouvé également une durée moyenne de 3 jours. Ces résultats sont similaires à ceux de Coulibaly $B$ [5], lequel trouve une durée moyenne d'hospitalisation de 4 jours dans $63,6 \%$ des cas, ainsi que Coulibaly MZ [14] qui trouve le même résultat. Nous avons enregistré $82,9 \%$ de guérison sans séquelles. Cependant, $12,4 \%$ des patients sont décédés et la cause des décès était le paludisme grave ( 6 cas) suivi de malnutrition aigüe sévère ( 5 cas), et de la septicémie (4 cas).

\section{Conclusion}

Les urgences pédiatriques représentent un maillon très important dans la prise en charge des enfants malades avec une fréquentation très élevée. Cependant les malades référés restent relativement peu nombreux malgré une pyramide sanitaire bien codifiée.

\section{Références}

1. Abdou $\mathrm{R} O$ et coll. Les urgences Pédiatriques au centre hospitalier de Libreville. Médecine d'Afrique Noire 2002 ; 49 (11): 475-80.

2. Chobli $M$ et coll. Créer un service d'aide médicale d'urgence dans un pays en voie de développement: luxe ou nécessité? 44ème congrès national d'anesthésie réanimation Société Française d'Anesthésie et de Réanimation (SFRAR) 2002.

3. Atanda H L, Porte J, Bon J C et coll. Mortalité et morbidité infantile sur une population d'un service médical à Pointe Noire. Médecine d'Afrique Noire 1991 ; 11: 24-35. 4. La politique sectorielle de santé et de population au Mali : ses premiers résultats (en ligne). Mali 2007 ; disponible sur HTM.

5. Coulibaly B. Urgences médicales dans le service de pédiatrie du CHU-GT. Thèse Med, Bamako 2006; №265.

6. Kourouma N. Les intoxications aigues accidentelles chez l'enfant : à propos de 89 cas au service de pédiatrie du CHU-GT. Thèse Med, Bamako 2003; №73.

7. Traoré 0 . Les prescriptions d'urgences en pédiatrie au CHU-GT. Thèse de Pharmacie, Bamako 2005; Nº71.

8. Sy O. Mortalité et morbidité dans le service de pédiatrie de l'hôpital Gabriel TOURE de 1999 à 2000. Thèse Med, Bamako 2001; N²7.

9. Bobossi-Seringne $B$ et Coll. Les fièvres prolongées de l'enfant : expérience du C.H.U de Bangui (Centrafrique). Médecine d'Afrique Noire 2002; 49(7): 346-350.

10. Daffé $H$. Urgences pédiatriques au service de pédiatrie de l'hôpital régional Nianankoro Fomba de Ségou. Thèse Med, Bamako 2006; №159.

11. Dan $V$ et coll. Prise en charges des urgences du nourrisson et de l'enfant: aspects actuels et perspectifs d'avenir au Centre National Hospitalier de Cotonou. Médecine d'Afrique Noire 1991; 38 (11): 752-759.

12. Coulibaly $S$. Fièvres prolongées chez l'enfant: étude clinique, étiologique, et évolutive dans le service de pédiatrie de l'hôpital Gabriel TOURE. Thèse Med, Bamako 2003; №13.

13. Costil J, Mothe J C, Chevalier J Y et coll. Détresse respiratoire de l'enfant. Encyclopédie Médico-Chirurgicale 1999; 10(1) : 6040.

14. Coulibaly M Z. Urgences pédiatriques à l'hôpital Gabriel TOURE. Thèse Med, Bamako 1988; N42.

\section{Liste des tableaux et figures :}


Tableau I : répartition des patients selon les caractéristiques socio-économiques.

\begin{tabular}{|c|c|c|}
\hline $\begin{array}{l}\text { Caractéristiques socio- } \\
\text { économiques }\end{array}$ & $\begin{array}{l}\text { Effectif } \\
(\mathrm{N}=234)\end{array}$ & $\%$ \\
\hline \multicolumn{3}{|l|}{ Age } \\
\hline $1-11$ mois & 120 & 51,3 \\
\hline $12-59$ mois & 74 & 31,6 \\
\hline $5-10$ ans & 14 & 6 \\
\hline Sup à 10 ans & 28 & 11,1 \\
\hline \multicolumn{3}{|l|}{ Sexe } \\
\hline Masculin & 154 & 66 \\
\hline Féminin & 80 & 34 \\
\hline \multicolumn{3}{|l|}{$\begin{array}{l}\text { Niveau d'instruction des } \\
\text { pères }\end{array}$} \\
\hline Primaire & 46 & 19,6 \\
\hline Secondaire & 28 & 12 \\
\hline Supérieur & 11 & 4,7 \\
\hline Coranique & 16 & 7,8 \\
\hline Non scolarisé & 133 & 58,8 \\
\hline \multicolumn{3}{|l|}{ Profession des pères } \\
\hline Fonctionnaire & 29 & 12,4 \\
\hline Paysan & 70 & 30 \\
\hline Ouvrier & 81 & 34,6 \\
\hline Commerçant & 46 & 19,7 \\
\hline Etudiant/Elève & 3 & 1,3 \\
\hline \multicolumn{3}{|l|}{$\begin{array}{l}\text { Niveau d'instruction des } \\
\text { mères }\end{array}$} \\
\hline Primaire & 54 & 23,1 \\
\hline secondaire & 20 & 8,5 \\
\hline Supérieur & 8 & 3,4 \\
\hline Coranique & 6 & 2,6 \\
\hline Non scolarisée & 146 & 62,4 \\
\hline \multicolumn{3}{|l|}{ Profession des mères } \\
\hline Femme au foyer & 199 & 85 \\
\hline Commerçante & 10 & 4,3 \\
\hline Fonctionnaire & 11 & 4,7 \\
\hline Etudiante/Elève & 14 & 6 \\
\hline
\end{tabular}

Tableau II: répartition des malades selon la structure de provenance et le motif de référence

\begin{tabular}{lcc}
\hline Structure de provenance & $\begin{array}{c}\text { Effectif } \\
(\mathbf{N = 2 3 4 )}\end{array}$ & $\%$ \\
\hline Hôpital & 15 & 6,4 \\
CSRéf & 126 & 53,8 \\
CSCom & 32 & 13,7 \\
Clinique privée & 26 & 11,1 \\
Cabinet privé & 35 & 15,0 \\
Motif de référence & & \\
Convulsion & 37 & 15,8 \\
Hyperthermie & 72 & 30,7 \\
Dyspnée & 105 & 44,8 \\
\hline
\end{tabular}

\begin{tabular}{lcc}
\hline Agitation & 7 & 3 \\
Coma & 12 & 5,1 \\
Diarrhée/Déshydratation & 20 & 8,5 \\
Anémie & 33 & 14,1 \\
OEdème & 7 & 3 \\
Intoxication & 4 & 1,7 \\
\hline
\end{tabular}

Tableau III : répartition des malades selon le diagnostic retenu

\begin{tabular}{lcc}
\hline Diagnostic retenu & Effectif & $\%$ \\
\hline Pathologies & 45 & 19,2 \\
bronchopulmonaires & & \\
Paludisme grave & 65 & 27,7 \\
Néphropathie & 3 & 1,3 \\
Déshydratation sévère / & 10 & 4,3 \\
gastroentérite & & \\
Méningite & 30 & 12,8 \\
Cardiopathie & 33 & 14,1 \\
Intoxication & 6 & 2,6 \\
Drépanocytose & 1 & 0,4 \\
Malnutrition & 10 & 4,3 \\
Infection ostéo-articulaire & 5 & 2,1 \\
Septicémie & 8 & 3,4 \\
Trisomie & 1 & 0,4 \\
Paludisme+méningite & 4 & 1,7 \\
Staphylococcie cutanée & 2 & 0,9 \\
Laryngite & 1 & 0,4 \\
Tuberculose pulmonaire & 1 & 0,4 \\
Pathologies Oncologiques & 7 & 2,9 \\
Corps étranger & 1 & 0,4 \\
Rétrovirose & 1 & 0,4 \\
Total & 234 & 100,0 \\
\hline & & \\
\hline
\end{tabular}

Tableau IV : répartition des patients selon la prise en charge

\begin{tabular}{lcc}
\hline Prise en charge & $\begin{array}{c}\text { Effectif } \\
\text { (N=234) }\end{array}$ & $\%$ \\
\hline Traitement & & \\
Amoxicilline injectable & 31 & 13,2 \\
Ceftriaxone injectable & 57 & 24,4 \\
Gentamycine injectable & 2 & 0,9 \\
Amoxicilline +acide & 52 & 22,2 \\
clavulanique & & \\
Ceftriaxone + gentamycine & 74 & 31,6 \\
Artesunate injectable & 101 & 43,0 \\
Durée du traitement & & \\
$\geq 3$ jours & 158 & 67,5 \\
4 - 7 jours & 56 & 23,9 \\
8- 14 jours & 16 & 6,8 \\
$>14$ jours & 4 & 1,7 \\
\hline
\end{tabular}




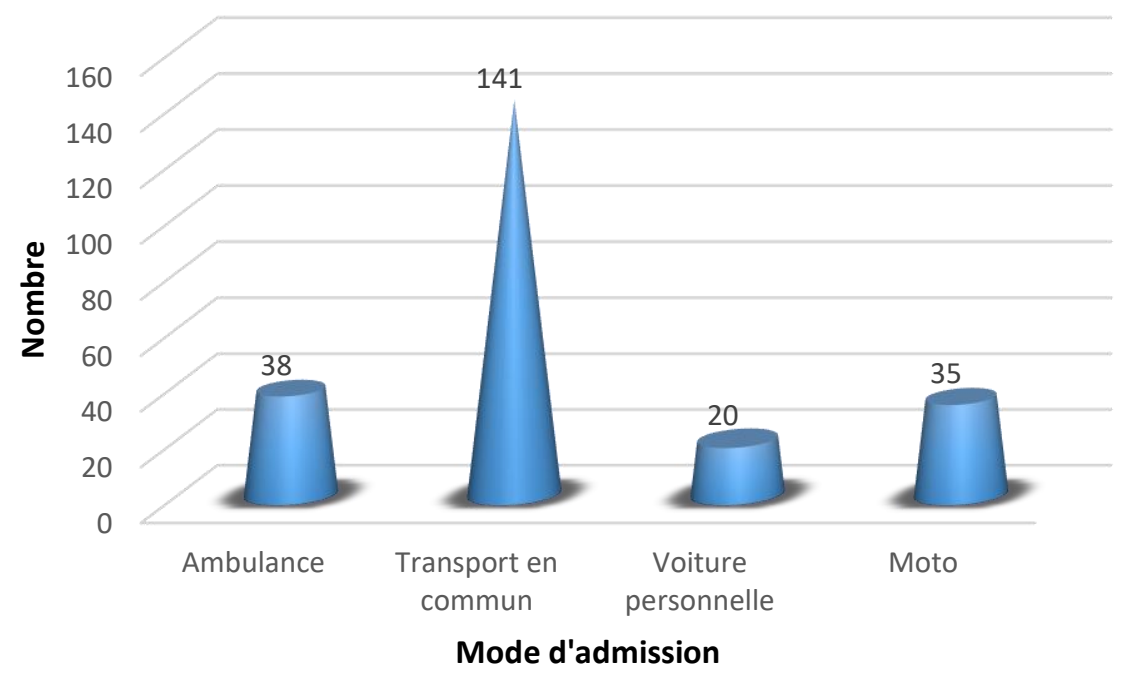

Figure 1 : répartition des malades selon le mode d'admission

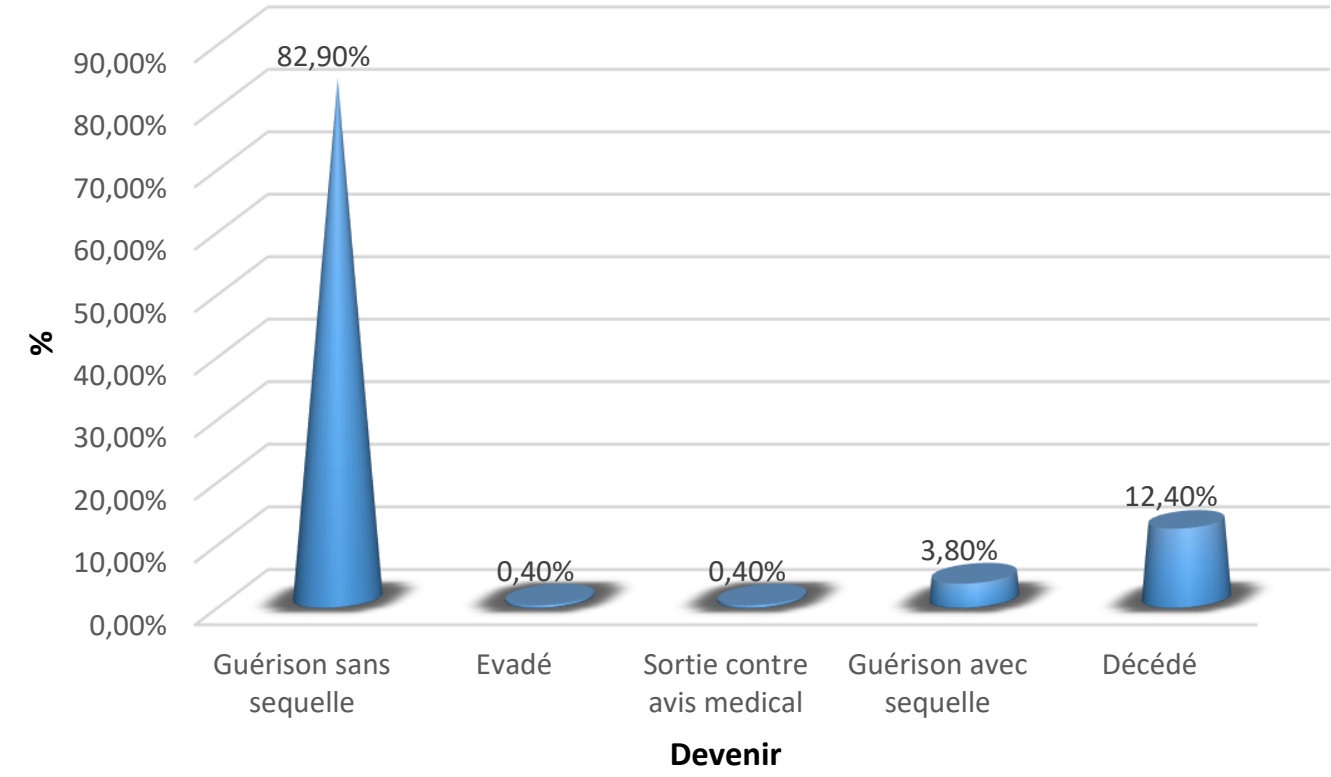

Figure 3 : répartition des malades selon le devenir 